\title{
Design of Attribute Control Chart based on Inverse Rayleigh Distribution under Type-I Censoring
}

\author{
C. Nanthakumar ${ }^{1 *}$, T. Kavitha ${ }^{2}$ \\ 1*PG \& Research Department of Statistics, Salem Sowdeswari College, Salem, India \\ ${ }^{2}$ PG \& Research Department of Statistics, Salem Sowdeswari College, Salem, India \\ *Corresponding Author: nanthakumarstat@gmail.com
}

Available online at: www.isroset.org

Received: 10/Oct/2017, Revised: 25/Oct/2017, Accepted: 20/Nov/2017, Published: 31/Dec/2017

\begin{abstract}
Control charts are one of the most common techniques that have been used to observe and control the process deviations in the industry. A control chart provides information about the performance or capability of a process. Lifetime considered as a quality characteristics for some products. Industries are routinely performing suitable life tests for their products. In life testing, censoring schemes are adopted to save time and cost of life test. Time censoring (type-I), product censoring (type-II) and hybrid censoring (Mixture of type-I and type-II censoring) are some of the censoring schemes employed in the life tests. The main objective of this paper is to design an life test based attribute control chart for inverse Rayleigh distribution under type-I censoring scheme. The performance of the control chart is studied using average run length $(A R L)$. Designed control chart is illustrated through numerical examples.
\end{abstract}

Keywords - Inverse Rayleigh distribution, attribute control chart, type-I censoring, average run length.

\section{INTRODUCTION}

The control charts are one of the most important statistical tool used in industries to monitor the production process for high quality of the products. Many control chart schemes have been developed to monitor the process for various situations. The control chart is a graph used to study how a process changes over time. A control chart always has a central line $(C L)$ for the average, an upper line for the upper control limit $(U C L)$ and a lower line for the lower control limit $(L C L)$. When the control statistic is plotted beyond the $L C L$ or $U C L$ limits, the process is declared as out of control. The process is said to be in control if the control statistic is inside the control limits. There are two type of control charts are available namely attribute and variable control charts. The attribute control charts are used to classify the nonconforming items from the conforming items. The variable control charts are used when the industrial data obtained from the measurement process.

Life time considered as a quality characteristics for some products. Monitoring the production process for such products is carried out by suitable life tests. Based on the information obtained from the life test the product may be classified as conforming or nonconforming. The testing of this type of products is time consuming as one has to wait for a long time until the test duration ends. In this situation, censoring scheme is an important and non-ignorable one. Type-I censoring, Type-II censoring and hybrid censoring are some of the censoring schemes employed in life testing. Under Type-I censoring, the life test is terminated at predetermined time $t$. During the life test, if the product failed before test termination time $t$, then the product may be classified as nonconforming product.

The attribute control chart such as $n p$ control chart is designed on the basis of the fraction nonconforming, which is obtained by assuming that the quality of interest follows the normal distribution. It may happen in the real life that the distribution of the quality of interest is not normal. In this case, the application of the existing control chart may mislead the industrial engineers and can cause the increment of the non-conforming items. Many authors in the literature have worked on attribute control charts including [1] - [9]. By exploring the literature, we note that there have been no studies on a control chart to incorporate a life test for a non normal distribution such as Inverse Rayleigh distribution under type-I censoring scheme.

Inverse Rayleigh distribution is one of the statistical distributions which attracted the attention of the researchers due to its applications in scientific and engineering related studies. This distribution has been applied widely as a life time distribution in reliability and life testing. It is a special case of inverse Weibull distribution. This distribution is used in situation of products which have initially more probable failures. 
In this paper, we will develop an attribute control chart based on inverse Rayleigh distribution under type-I censoring scheme. We will find the control chart co-efficient and discuss the behaviour of average run length of the proposed control chart. The designing of the proposed control chart is presented when the scale parameter is shifted. The application of the proposed chart is given with the help of simulated data. A brief introduction about the Inverse Rayleigh distribution is given in Section 2. Designing of the control chart for this distribution is discussed in Section 3. A numerical illustration is given in Section 4. Finally the conclusions are given in Section 5.

\section{INVERSE RAYLEIGH DISTRIBUTION}

[10] Defined inverse Rayleigh distribution for applying in reliability and survived analysis since it has monotone failure rate. [11] Mentioned that the distribution of lifetimes of several types of experimental units can be approximated by the inverse Rayleigh distribution.

Let $T$ be the lifetime of the product. Which is distributed according to the inverse Rayleigh distribution with scale parameter $(\theta)$, then the probability density function of $T$ is given by

$f(t ; \theta)=\left(\frac{2 \theta}{t^{3}}\right) e^{\left(-\frac{\theta}{t^{2}}\right)} ; t>0, \theta>0$

The cumulative distribution function of the inverse Rayleigh distribution $(\theta)$ is given by

$F(t ; \theta)=e^{\left(-\frac{\theta}{t^{2}}\right)} ; t>0, \theta>0$

The mean life of a product for this distribution is given as

$\mu=\sqrt{\pi \theta}$

[11] - [14] studied more on the inverse Rayleigh distribution.

\section{DESIGN OF CONTROL CHART BASED ON INVERSE}

\section{RAYLEIGH DISTRIBUTION}

We propose the following $n p$ control chart for inverse Rayleigh distribution under type-I censoring scheme based on the number of products for each subgroup:

Step 1 Select a set of $n$ products randomly from the production process.
Step 2 Conduct the life test on the selected items considering $t$ as the test termination time. Observe the number of failed items $(D$, say).

Step 3 Terminate the life test after reached at time $t$.

Step 4 Declare the process as out of control if $D>\mathrm{UCL}$ or $D<$ LCL. Declare the process as in control if $\mathrm{LCL} \leq D \leq \mathrm{UCL}$.

Note that the above is called as $n p$ control chart because the number of failures $(D)$ rather than the fraction nonconforming $(p)$ is plotted. The random variable $D$ follows a binomial distribution with parameters $n$ and $p_{0}$ when process is in control, where $p_{0}$ is the probability that an item is failed before time $t$. Therefore, the control limits for in control process are given as follows:

$U C L=n p_{0}+k \sqrt{n p_{0}\left(1-p_{0}\right)}$

$L C L=\max \left[0, n p_{0}-k \sqrt{n p_{0}\left(1-p_{0}\right)}\right]$

Where, $k$ is the coefficient of the control limits. The fraction nonconforming in control process $\left(p_{0}\right)$ may be obtained from equation (2) by

$p_{0}=e^{\left(-\frac{\theta_{0}}{t^{2}}\right)}=e^{\left(-\frac{1}{a^{2} \pi}\right)}$

In practice, probability $p_{0}$ is usually unknown, therefore, the control limit for the practical application are given as

$U C L=\bar{D}+k \sqrt{\bar{D}\left(1-\frac{\bar{D}}{n}\right)}$

$L C L=\max \left[0, \bar{D}-k \sqrt{\bar{D}\left(1-\frac{\bar{D}}{n}\right)}\right]$

Where $\bar{D}$ is the average number of failures over the subgroups.

The probability of declaring as in control for the proposed control chart is given as follows:

$p_{i n}^{0}=P\left(L C L \leq D \leq U C L \mid p_{0}\right)$

$p_{i n}^{0}=\sum_{d=[L C L]+1}^{[U C L]}\left(\begin{array}{l}n \\ d\end{array}\right) p_{0}^{d}\left(1-p_{0}\right)^{n-d}$ 
The performance of the control chart is usually evaluated by average run length. The $A R L$ for in control process as follows:

$$
A R L_{0}=\frac{1}{1-\left[\sum_{d=[L C L]+1}^{U C L}\left(\begin{array}{l}
n \\
d
\end{array}\right) p_{0}^{d}\left(1-p_{0}\right)^{n-d}\right]}
$$

\section{1. $A R L$ WHEN PROCESS MEAN IS SHIFTED}

Suppose now that the process mean is shifted from $\mu_{0}$ to $\mu_{1}$. Then the probability in (5) becomes

$$
p_{1}=e^{\left(-\frac{\theta_{1}}{t^{2}}\right)}=e^{-\frac{1}{a^{2} \pi}\left(\frac{\mu_{1}}{\mu_{0}}\right)^{2}}
$$

If we consider the mean shift such as $\mu_{1}=f \mu_{0}$ for a constant $f$, then equation (9) is rewritten as

$$
p_{1}=e^{-\left(\frac{f^{2}}{a^{2} \pi}\right)}
$$

Now, the probability that the process is declared to be in control when the process has shifted to $\mu_{l}$ is given by

$$
\begin{aligned}
& p_{\text {in }}^{1}=P\left(L C L \leq D \leq U C L \mid p_{1}\right) \\
& p_{\text {in }}^{1}=\sum_{d=[L C L]+1}^{[U C L]}\left(\begin{array}{l}
n \\
d
\end{array}\right) p_{1}^{d}\left(1-p_{1}\right)^{n-d}
\end{aligned}
$$

The $A R L$ for the shifted process is given as follows:

$$
A R L_{1}=\frac{1}{1-\left[\sum_{d=[L C L]+1}^{U C L}\left(\begin{array}{l}
n \\
d
\end{array}\right) p_{1}^{d}\left(1-p_{1}\right)^{n-d}\right]}
$$

We used the following algorithm to complete the tables for the proposed control chart.

(1) Specify the values of $A R L$, say $r_{0}$ and sample size $n$.

(2) Determine the values of control chart parameters and truncated time constant $a$ for which the ARL from equation (8) is close to $r_{0}$.

(3) Use the values of control chart parameters obtained in step 2 to find the $A R L_{l}$ according to shift constant $f$ using equation (12).
We determine the control chart parameters and $A R L_{l}$ for various values of $r_{0}$, and $n$ from Tables $1 \& 2$, we note that the decreasing tend in $A R L_{S}$ as the shift constant $f$ decreases.

Table 1 ARLs for the proposed chart for $\mathrm{n}=20$ when the mean shifted

\begin{tabular}{|c|r|r|r|}
\hline & \multicolumn{1}{|c|}{$r_{0}=200$} & \multicolumn{1}{|c|}{$r_{0}=400$} & \multicolumn{1}{c|}{$r_{0}=500$} \\
\hline $\mathrm{a}$ & 0.595 & 0.5231 & 0.5109 \\
\hline $\mathrm{k}$ & 2.567 & 2.774 & 2.742 \\
\hline $\mathrm{UCL}$ & 14 & 12 & 12 \\
\hline $\mathrm{LCL}$ & 2 & 0 & 0 \\
\hline Shift $(f)$ & \multicolumn{1}{|c}{$A R L$} & \multicolumn{1}{|c|}{$A R L$} & \multicolumn{1}{c|}{$A R L$} \\
\hline 1.00 & 200.01 & 400.04 & 500.29 \\
\hline 0.90 & 69.04 & 60.01 & 91.11 \\
\hline 0.80 & 14.51 & 11.19 & 14.88 \\
\hline 0.70 & 4.40 & 3.35 & 3.96 \\
\hline 0.60 & 1.96 & 1.59 & 1.72 \\
\hline 0.50 & 1.25 & 1.12 & 1.15 \\
\hline 0.40 & 1.04 & 1.01 & 1.02 \\
\hline 0.30 & 1.00 & 1.00 & 1.00 \\
\hline 0.20 & 1.00 & 1.00 & 1.00 \\
\hline 0.10 & 1.00 & 1.00 & 1.00 \\
\hline
\end{tabular}

Table 2 ARLs for the proposed chart for $\mathrm{r}_{0}=300$ when the mean shifted

\begin{tabular}{|c|r|r|r|}
\hline & \multicolumn{1}{|c|}{$n=25$} & \multicolumn{1}{|c|}{$n=39$} & \multicolumn{1}{c|}{$n=43$} \\
\hline $\mathrm{a}$ & 0.5134 & 0.6498 & 0.4446 \\
\hline $\mathrm{k}$ & 2.634 & 2.84 & 2.705 \\
\hline $\mathrm{UCL}$ & 14 & 27 & 16 \\
\hline $\mathrm{LCL}$ & 1 & 9 & 1 \\
\hline Shift $(f)$ & $A R L$ & $A R L$ & \multicolumn{1}{|c|}{$A R L$} \\
\hline 1.00 & 300.04 & 300.02 & 300.15 \\
\hline 0.90 & 52.06 & 50.85 & 19.06 \\
\hline 0.80 & 8.49 & 7.78 & 2.85 \\
\hline 0.70 & 2.52 & 2.31 & 1.21 \\
\hline 0.60 & 1.31 & 1.25 & 1.01 \\
\hline 0.50 & 1.04 & 1.03 & 1.00 \\
\hline 0.40 & 1.00 & 1.00 & 1.00 \\
\hline 0.30 & 1.00 & 1.00 & 1.00 \\
\hline 0.20 & 1.00 & 1.00 & 1.00 \\
\hline 0.10 & 1.00 & 1.00 & 1.00 \\
\hline
\end{tabular}

\subsection{ARL WHEN SCALE PARAMETER IS SHIFTED}

Suppose now that the scale parameter is shifted from $\theta_{0}$ to $\theta_{l}$. Then the probability in (5) becomes

$p_{2}=e^{\left(-\frac{\theta_{1}}{t^{2}}\right)}$

If we consider the scale parameter shift such as $\theta_{l}=c \theta_{0}$ for a constant $c$, then equation (13) is rewritten as

$p_{2}=e^{\left(-\frac{c}{a^{2} \pi}\right)}$

Now, the probability that the process is declared to be in control when the process has shifted to $\theta_{1}$ is given by 


$$
p_{\text {in }}^{2}=P\left(L C L \leq D \leq U C L \mid p_{2}\right)
$$

The $A R L$ for the shifted process is given as follows:

$$
A R L_{1}=\frac{1}{1-\left[\sum_{d=[L C L]+1}^{U C L}\left(\begin{array}{l}
n \\
d
\end{array}\right) p_{2}^{d}\left(1-p_{2}\right)^{n-d}\right]}
$$

We used the following algorithm to complete the tables for the proposed control chart.

(1) Specify the values of $A R L$, say $r_{0}$ and sample size $n$.

(2) Determine the values of control chart parameters and truncated time constant $a$ for which the $A R L$ from equation (8) is close to $r_{0}$.

(3) Use the values of control chart parameters obtained in step 2 to find the $A R L_{l}$ according to shift constant $c$ using equation (16).

We determine the control chart parameters and $A R L_{1}$ for various values of $r_{0}$, and $n$ from Tables $3 \& 4$, we note that the decreasing tend in $\mathrm{ARL}_{\mathrm{S}}$ as the shift constant $c$ decreases.

Table 3 ARLs for the proposed chart for $n=20$ when the scale parameter shifted

\begin{tabular}{|c|r|r|r|}
\hline & $r_{0}=200$ & $r_{0}=400$ & \multicolumn{1}{c|}{$\begin{array}{l}r_{0}= \\
\end{array}$} \\
\hline $\mathrm{a}$ & 0.595 & 0.5231 & 0.5109 \\
\hline $\mathrm{k}$ & 2.567 & 2.774 & 2.742 \\
\hline $\mathrm{UCL}$ & 14 & 12 & 12 \\
\hline $\mathrm{LCL}$ & 2 & 0 & 0 \\
\hline Shift $(c)$ & $A R L$ & $A R L$ & $A R L$ \\
\hline 1.00 & 200.01 & 400.04 & 500.29 \\
\hline 0.90 & 148.04 & 157.20 & 246.19 \\
\hline 0.80 & 62.89 & 54.00 & 81.42 \\
\hline 0.70 & 24.76 & 19.63 & 27.38 \\
\hline 0.60 & 10.33 & 7.87 & 10.14 \\
\hline 0.50 & 4.73 & 3.59 & 4.28 \\
\hline 0.40 & 2.45 & 1.93 & 2.14 \\
\hline 0.30 & 1.49 & 1.27 & 1.33 \\
\hline 0.20 & 1.11 & 1.04 & 1.05 \\
\hline 0.10 & 1.01 & 1.00 & 1.00 \\
\hline
\end{tabular}

Table 4 ARLs for the proposed chart for $r_{0}=300$ when the scale parameter shifted

\begin{tabular}{|c|r|r|r|}
\hline & \multicolumn{1}{|c|}{$n=25$} & $n=39$ & \multicolumn{1}{c|}{$n=43$} \\
\hline $\mathrm{a}$ & 0.5134 & 0.6498 & 0.4446 \\
\hline $\mathrm{k}$ & 2.634 & 2.84 & 2.705 \\
\hline $\mathrm{UCL}$ & 14 & 27 & 16 \\
\hline $\mathrm{LCL}$ & 1 & 9 & 1 \\
\hline Shift $(c)$ & \multicolumn{1}{|c}{$A R L$} & \multicolumn{1}{|c}{$A R L$} & \multicolumn{1}{c|}{$A R L$} \\
\hline 1.00 & 300.04 & 300.02 & 300.15 \\
\hline 0.90 & 146.92 & 152.82 & 71.90 \\
\hline 0.80 & 46.37 & 45.04 & 16.64 \\
\hline 0.70 & 15.42 & 14.34 & 5.01 \\
\hline 0.60 & 5.89 & 5.37 & 2.09 \\
\hline 0.50 & 2.69 & 2.46 & 1.25 \\
\hline 0.40 & 1.53 & 1.44 & 1.03 \\
\hline 0.30 & 1.12 & 1.09 & 1.00 \\
\hline 0.20 & 1.01 & 1.01 & 1.00 \\
\hline 0.10 & 1.00 & 1.00 & 1.00 \\
\hline
\end{tabular}

\section{ILLUSTRATION}

Suppose that the lifetime of the products follows the inverse Rayleigh distribution. Suppose that the target average life of the product is $\mu_{0}=1000$ hours, $a=0.5231, r_{0}=400$ and $n=20$. Then from equation (5), we get $p_{0}=0.3125$. Also, from Table 1 we obtain the $k=2.774$, LCL $=0$ and $\mathrm{UCL}=12$. Therefore the designed control chart as follows:

Step 1: Take a sample of size 20 at each subgroup and put them on the life test during $t=523$ hours. Count the number of failed items $(D)$ during the test.

Step 2: Declare the process as in control if $0 \leq D \leq 12$ and as out of control, otherwise.

\section{Simulation Study}

In this section, the application of the proposed chart is discussed with the help of simulated data. The data are generated using the inverse Rayleigh distribution with an average lifetime $\mu_{0}=1000$ hours. Let $n=20$ and $r_{0}=200$. It is declared that the process is in control when $\mu_{0}=1000$ hours and $f=1$. The first 15 observations of subgroup size 20 are generated using in control parameters. Now, suppose that the process has shifted due to the shift in the mean of inverse Rayleigh distribution. The shift constant $f$ is taken as 0.7 . The next 15 observations are generated with shifted mean when $f=0.7$.

Let life test termination time $t=595$ hours. The number of failures $D$ are counted and reported in Table 5 for each subgroup. The average number of failures $\bar{D}$ is computed. The UCL $=14$ and $\mathrm{LCL}=2$ is for simulated data. The data are plotted in Figure 1. 
Table 5 Simulated Data

\begin{tabular}{|c|c|c|c|c|c|c|c|c|c|c|c|c|c|c|c|c|c|c|c|c|c|}
\hline \multirow{2}{*}{$\begin{array}{l}\text { S. } \\
\text { No. }\end{array}$} & \multicolumn{20}{|c|}{ Sample } & \multirow{2}{*}{ D } \\
\hline & 1 & 2 & 3 & 4 & 5 & 6 & 7 & 8 & 9 & 10 & 11 & 12 & 13 & 14 & 15 & 16 & 17 & 18 & 19 & 20 & \\
\hline 1 & 834 & 2706 & 444 & 699 & 432 & 710 & 615 & 323 & 627 & 863 & 751 & 608 & 448 & 355 & 2596 & 1994 & 365 & 414 & 749 & 569 & 8 \\
\hline 2 & 915 & 1277 & 1328 & 634 & 317 & 653 & 460 & 588 & 715 & 686 & 640 & 469 & 2461 & 1460 & 466 & 564 & 1131 & 491 & 697 & 710 & 7 \\
\hline 3 & 1077 & 697 & 715 & 375 & 341 & 325 & 517 & 585 & 627 & 627 & 275 & 889 & 2870 & 6221 & 832 & 803 & 1689 & 510 & 1850 & 1381 & 7 \\
\hline 4 & 1472 & 1036 & 894 & 831 & 403 & 391 & 582 & 1812 & 282 & 1884 & 606 & 425 & 1401 & 946 & 688 & 845 & 453 & 214 & 761 & 451 & 8 \\
\hline 5 & 1099 & 564 & 517 & 413 & 689 & 1131 & 381 & 2668 & 1331 & 407 & 874 & 653 & 301 & 412 & 1083 & 929 & 542 & 763 & 489 & 637 & 9 \\
\hline 6 & 602 & 373 & 580 & 588 & 429 & 507 & 441 & 2457 & 650 & 810 & 597 & 369 & 671 & 3709 & 695 & 510 & 1069 & 431 & 927 & 546 & 10 \\
\hline 7 & 605 & 525 & 557 & 662 & 404 & 361 & 562 & 812 & 634 & 1527 & 2703 & 1598 & 727 & 882 & 771 & 701 & 305 & 950 & 564 & 574 & 8 \\
\hline 8 & 746 & 2558 & 480 & 297 & 736 & 521 & 628 & 1851 & 1585 & 241 & 449 & 899 & 1131 & 353 & 760 & 686 & 349 & 356 & 1386 & 724 & 8 \\
\hline 9 & 731 & 508 & 583 & 794 & 472 & 332 & 614 & 374 & 406 & 519 & 707 & 2231 & 565 & 852 & 352 & 1214 & 1062 & 637 & 1434 & 303 & 10 \\
\hline 10 & 463 & 447 & 705 & 1125 & 463 & 578 & 1968 & 473 & 1253 & 872 & 471 & 392 & 578 & 491 & 2612 & 651 & 1285 & 444 & 220 & 1674 & 11 \\
\hline 11 & 957 & 637 & 4584 & 576 & 690 & 356 & 1154 & 1443 & 621 & 1123 & 388 & 967 & 725 & 989 & 1489 & 371 & 1119 & 987 & 458 & 317 & 6 \\
\hline 12 & 1654 & 1324 & 4355 & 1439 & 596 & 605 & 3664 & 299 & 403 & 3495 & 5609 & 715 & 536 & 451 & 2065 & 1454 & 413 & 1081 & 678 & 1376 & 5 \\
\hline 13 & 561 & 510 & 759 & 712 & 396 & 496 & 696 & 655 & 595 & 1664 & 1010 & 471 & 1745 & 614 & 1394 & 852 & 403 & 1607 & 536 & 338 & 7 \\
\hline 14 & 709 & 2076 & 451 & 1591 & 2615 & 660 & 1011 & 2951 & 936 & 978 & 2712 & 489 & 491 & 989 & 523 & 618 & 1016 & 1077 & 507 & 646 & 5 \\
\hline 15 & 323 & 1123 & 1937 & 580 & 1377 & 531 & 444 & 1381 & 280 & 536 & 779 & 644 & 945 & 917 & 2840 & 592 & 1084 & 856 & 534 & 405 & 9 \\
\hline 16 & 928 & 340 & 298 & 385 & 292 & 684 & 365 & 348 & 287 & 756 & 513 & 618 & 871 & 510 & 885 & 1125 & 658 & 767 & 478 & 344 & 11 \\
\hline 17 & 300 & 697 & 403 & 325 & 444 & 452 & 905 & 1411 & 384 & 254 & 6589 & 1076 & 278 & 235 & 485 & 368 & 292 & 349 & 230 & 667 & 14 \\
\hline 18 & 283 & 550 & 856 & 262 & 512 & 714 & 523 & 686 & 261 & 552 & 188 & 1161 & 649 & 480 & 458 & 1799 & 377 & 319 & 414 & 334 & 14 \\
\hline 19 & 306 & 1802 & 212 & 561 & 1584 & 379 & 439 & 803 & 1005 & 423 & 886 & 458 & 226 & 589 & 727 & 750 & 657 & 373 & 1600 & 819 & 11 \\
\hline 20 & 421 & 384 & 222 & 435 & 264 & 1165 & 515 & 544 & 281 & 560 & 1207 & 821 & 495 & 532 & 455 & 429 & 448 & 310 & 419 & 483 & 17 \\
\hline 21 & 604 & 630 & 1563 & 163 & 759 & 269 & 434 & 180 & 1068 & 506 & 253 & 702 & 680 & 256 & 1509 & 246 & 454 & 269 & 730 & 877 & 10 \\
\hline 22 & 511 & 545 & 738 & 393 & 599 & 836 & 1578 & 220 & 930 & 694 & 836 & 4942 & 230 & 802 & 192 & 1660 & 381 & 363 & 286 & 332 & 10 \\
\hline 23 & 272 & 730 & 287 & 1622 & 681 & 450 & 636 & 784 & 256 & 277 & 405 & 563 & 652 & 342 & 3690 & 360 & 462 & 304 & 359 & 1460 & 12 \\
\hline 24 & 320 & 501 & 449 & 437 & 372 & 284 & 433 & 641 & 1367 & 777 & 262 & 1307 & 248 & 396 & 218 & 1073 & 403 & 5206 & 263 & 912 & 13 \\
\hline 25 & 447 & 601 & 530 & 262 & 384 & 286 & 1082 & 890 & 190 & 435 & 814 & 350 & 360 & 317 & 368 & 253 & 504 & 1053 & 410 & 1226 & 14 \\
\hline 26 & 4496 & 1826 & 583 & 170 & 329 & 547 & 831 & 340 & 1249 & 1750 & 333 & 745 & 499 & 201 & 1075 & 205 & 641 & 777 & 460 & 410 & 11 \\
\hline 27 & 290 & 956 & 464 & 327 & 4838 & 1274 & 903 & 258 & 366 & 410 & 5776 & 480 & 720 & 720 & 393 & 8549 & 686 & 517 & 570 & 1216 & 10 \\
\hline 28 & 412 & 726 & 731 & 270 & 1375 & 509 & 537 & 252 & 850 & 490 & 384 & 594 & 474 & 315 & 754 & 444 & 630 & 235 & 307 & 355 & 14 \\
\hline 29 & 452 & 601 & 486 & 216 & 477 & 891 & 293 & 559 & 304 & 867 & 803 & 745 & 417 & 277 & 961 & 6601 & 576 & 2279 & 699 & 449 & 11 \\
\hline 30 & 968 & 500 & 629 & 930 & 538 & 2093 & 389 & 483 & 713 & 774 & 543 & 627 & 369 & 939 & 340 & 554 & 1358 & 257 & 286 & 622 & 10 \\
\hline
\end{tabular}

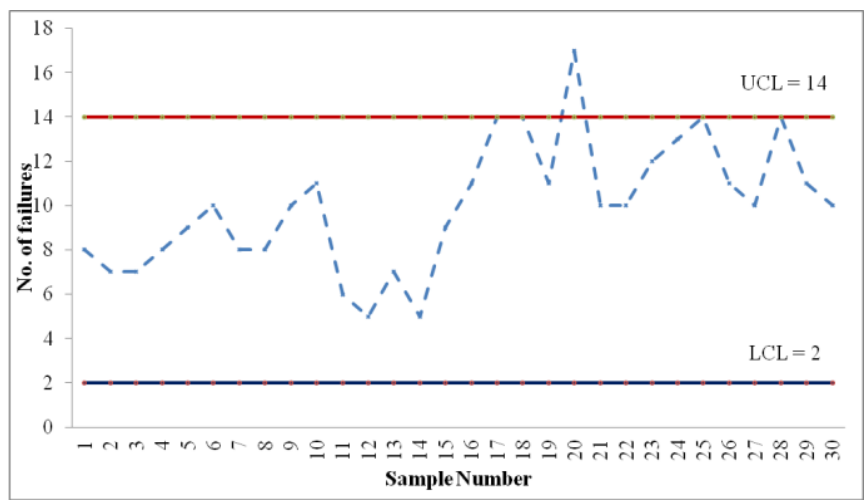

Figure 1. Control chart for simulation data.
From Figure 1, it can be observed that the proposed chart shows that shift at $20^{\text {th }}\left(5^{\text {th }}\right.$ observation after the shift) observation while tabulated ARL is 4.40. So, the proposed chart efficiently detects the shift in the process.

\section{CONCLUSION}

In this paper, a new attribute control chart is developed for the inverse Rayleigh distribution under type-I censoring. The new control chart is quite flexible and can be used to monitor the lifetimes of quality products. The tables are presented for the industrial use and explained with the help of simulated data. The performance of the new control chart is reported in terms of $A R L s$ according to various shift parameters. It may be noted that if the type-I censoring scheme is employed to carry out the life test, implementation of the sampling inspection 
will reduce the time and cost of conducting the life test. The proposed attribute control chart can be extended for some other distributions as a future research.

\section{ACKNOWLEDGEMENT}

The authors are thankful to the editor and the referees for their useful suggestions to improve the quality of paper.

\section{REFERENCES}

[1] E. K. Epprecht, A. F. B. Costa, F. C. T. Mendes, "Adaptive control charts for attributes", IIE Transction, Vol. 35, No. 6, pp. 567-582, 2003.

[2] A. F. B. Costa, M. A. Rahim, "Joint $X$ bar and $R$ charts with two stages samplings", Quality and Reliability Engineering International, Vol. 20, No. 7, pp. 699-708, 2004.

[3] L. F. Hsu, "Note on Design of Double and Triple Sampling Control Charts Using Genetic Algorithms", International Journal of Production Research, Vol. 42, No. 5, pp. 10431047, 2004.

[4] L. F. Hsu, "Note on Construction of Double Sampling $s$ Control Charts for Agile Manufacturing", Quality and Reliability Engineering International, Vol. 23, pp. 269-272, 2007.

[5] Z. Wu, H. Luo, X. Zhang, "Optimal $n p$ control chart with curtailment", European Journal of Operational Research, Vol. 174, pp. 1723-1741, 2006.

[6] Z. Wu, Q. Wang, "An $n p$ control chart using double inspections", Journal of Applied Statistics, Vol. 34, No. 7, pp. 843-855, 2007.

[7] E. P. Barbosa, S. Joekes, "An improved attribute control chart for monitoring non-conforming proportion in high quality process", Control Engineering Practice, Vol. 21, pp. 407-412, 2013.

[8] Mohammad Aslam, Chi-Hyuck Jun, "Attribute Control Charts for the Weibull Distribution under Truncated Life Tests", Quality Engineering, Vol. 27, No. 3, pp.283-288, 2015.

[9] Muhammad Aslam, Nasrullah Khan, Chi-Hyuck Jun, "A control chart for time truncated life tests using Pareto distribution of second kind", Journal of Statistical Computation and Simulation, Vol. 86, No. 11, pp. 21132122, 2016.

[10] V. N. Treyer, "Doklady Acad", Nauk, Belorus, U.S.S.R, 1964.

[11] R. Gh. Voda, "On the inverse Rayleigh Random Variable", Rep. Stat. Apph. Res. Juse, Vol. 19, No. 4, pp. 15-21, 1972.

[12] M. K. Gharrap, "Comparison of estimators of location measures of an inverse Rayleigh distribution", The Egyptian Statistical Journal, Vol. 37, No. 2, pp. 295-309, 1993.

[13] S. P. Mukarjee, S. S. Maitim, "A percentile Estimator of the Inverse Rayleigh Parameter", IAPQR Transactions, Vol.21, pp. 63-65, 1996.

[14] A. Soliman, E. A. Amin, A. A. Abd-Ei Aziz, "Estimation and Prediction from Inverse Rayleigh Distribution based on Lower Record Values", Applied Mathematical Sciences, Vol. 4, No. 62, pp. 3057-3066, 2010.

\section{AUTHORS PROFILE}

Dr. C. Nanthakumar pursed M.Phil. and Ph.D. Statistics from Madras University, Chennai in 1986 \& 2005. He is currently working as Associate Professor in PG \& Research Department of Statistics from Salem Sowdeswari College, Salem since 1986. He has published more than 10 research papers in reputed international journals including Thomson Reuters (SCI \& Web of Science). His main research work focuses on Statistical Quality Control, Operation Research and Survival Analysis. He has 27 years of teaching experience and 12 years of research experience.

Mrs. T. Kavitha is doctoral student in the PG \& Research Department of Statistics at Salem Sowdeswari College, Salem. Tamil Nadu. 